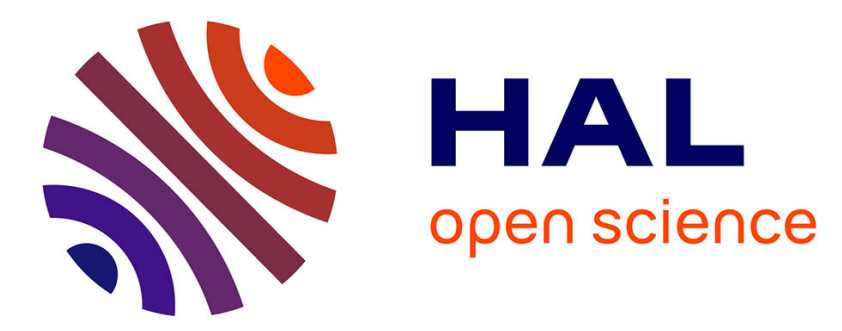

\title{
Dynamique de la population de truite commune (Salmo trutta) d'un ruisseau breton (France): les géniteurs sédentaires
}

\author{
Gérard Maisse, Jean-Luc J.-L. Baglinière, Pierre-Yves Le Bail
}

\section{To cite this version:}

Gérard Maisse, Jean-Luc J.-L. Baglinière, Pierre-Yves Le Bail. Dynamique de la population de truite commune (Salmo trutta) d'un ruisseau breton (France): les géniteurs sédentaires. Hydrobiologia, 1987, 148, pp.123-130. 10.1007/BF00008397 . hal-02728103

\section{HAL Id: hal-02728103 \\ https://hal.inrae.fr/hal-02728103}

Submitted on 2 Jun 2020

HAL is a multi-disciplinary open access archive for the deposit and dissemination of scientific research documents, whether they are published or not. The documents may come from teaching and research institutions in France or abroad, or from public or private research centers.
L'archive ouverte pluridisciplinaire HAL, est destinée au dépôt et à la diffusion de documents scientifiques de niveau recherche, publiés ou non, émanant des établissements d'enseignement et de recherche français ou étrangers, des laboratoires publics ou privés. 


\title{
Dynamique de la population de Truite commune (Salmo trutta) d'un ruisseau breton (France): les géniteurs sédentaires
}

\author{
G. Maisse ${ }^{1}$, J. L. Bagliniere ${ }^{2}$ \& P. Y. Le Bail ${ }^{1}$ \\ ${ }^{1}$ INRA - Laboratoire de Physiologie des Poissons, 35042 Rennes, Cédex; \\ ${ }^{2}$ INRA - Laboratoire d'Ecologie Hydrobiologique, 35042 Rennes, Cédex
}

Keywords: fish, trout, population dynamic, spawners, streams

\begin{abstract}
Population dynamics of brown trout (Salmo trutta) in a breton stream (France): The non-migrant spawners.

The biological factors of the population dynamic of brown trout in a tributary of the Scorff river, have been studied during 4 years. The characteristics of the spawners, present in the stream before the spawning migration from the main river, are described. These spawners were born in the stream and stayed there during their entire life-span. Among mature fish the sex ratio was always in favour of males. During the third and the fourth years of life, annual mortality rate was $70 \%$ for males and $50 \%$ for females. $1+$ spawners were these which had the best $0+$ growth rate. A majority of males first matured at $1+$ years of age. On an average, $1+$ females were responsible for $25 \%$ of the total reproductive potential. The number of $3+$ and $4+$ females seemed to depend on the fishing effort.
\end{abstract}

\section{Introduction}

Depuis [1974], la population de Truite commune (Salmo trutta) du Scorff (rivière de Bretagne sud) est étudiée. Les observations réalisées ont permis de mettre en évidence, pour cette espèce, le rôle primordial des affluents pour la fraie et le recrutement en juvéniles de la population de la rivière principale. [Euzenat \& Fournel, 1976; Baglinière et al., 1979a; Nihouarn, 1983.] Cependant, si ces études ainsi que celles effectuées dans d'autres régions [Libosvarsky, 1967; Huet \& Timmermans, 1979; Prouzet, 1981] décrivent bien les populations migrantes (géniteurs et juvéniles), le rôle de la population en place, avant la migration de fraie des géniteurs de la rivière principale, est peu étudié. Dans le cadre d'une étude de la dynamique de la population de Truite du ruisseau de Kernec, affluent du Scorff, trois aspects sont abordés:
- les géniteurs sédentaires de l'affluent;

- les géniteurs du Scorff frayant dans l'affluent;

- les juvéniles produits dans l'affluent et leur devenir.

Les résultats présentés dans cet article concernent le premier volet de cette étude.

\section{Matériél et méthodes}

\section{Le milieu}

Le ruisseau de Kernec est un affluent rive droite de la zone aval du Scorff (Fig. 1). D'une longueur d'environ $6 \mathrm{~km}$ et d'une largeur maximal de $3 \mathrm{~m}$, son cours traverse des terrains granitiques. Les parties amont $\left(2660 \mathrm{~m}^{2}\right)$ et aval $\left(1950 \mathrm{~m}^{2}\right)$ se caractérisent par une forte pente (jusqu'à $9,5 \%$ ) et une succession de zones de courant sur fond de gravier. 


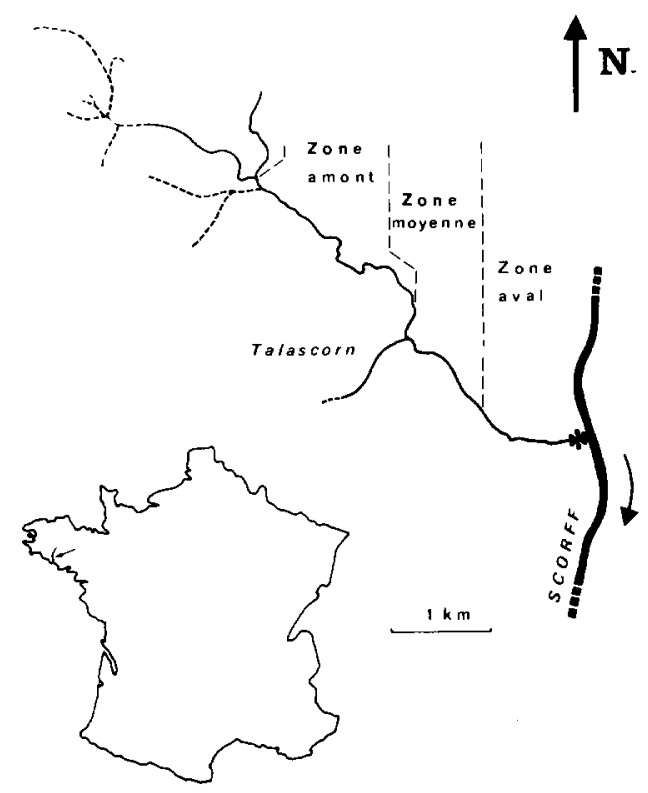

Fig. 1. Situation des zones d'études sur le ruisseau de Kernec $\star$ Dispositif de capture (montaison et avalaison).

La partie moyenne $\left(2100 \mathrm{~m}^{2}\right)$, à pente plus faible $(4 \%)$ est à courant plus lent, sur fond sableux, avec des sous berges importantes.

Les caractéristiques de l'habitat ont été décrites par Nihouarn [1983].

\section{Les inventaires}

De 1980 à 1983 inclus, le ruisseau de Kernec à été inventorié par pêche électrique (méthode de De Lury, avec 2 passages) sur $3850 \mathrm{~m}$ en amont de la station de piégeage (montaison et avalaison) située à $50 \mathrm{~m}$ de sa confluence avec le Scorff. Ces inventaires ont toujours eu lieu la 2ème semaine d'octobre. L'efficacité des pêches (nombre de poissons capturés/nombre estimé de poissons) sur les géniteurs à été de $99,9 \%$ chaque année [populations estimées à l'aide de la formule de Seber et Lecren, 1967] malgré des variations de conditions hydriques qui ont affecté principalement la capturabilité des $0+$ et des petits $1+$. La répartition des classes d'âge a été faite par scalimétrie en liaison avec l'étude de l'histogramme de fréquence de tailles (longueur fourche en $\mathrm{mm}$ ).
Le sexage

A l'époque des inventaires aucun dimorphisme sexuel n'a pu être utilisé pour sexer correctement les géniteurs à l'exception de quelques mâles âgés. Les femelles en vitellogenèse ont donc été mises en évidence à l'aide du test d'immunoagglutination de la vitellogénine plasmatique [Le Bail et Breton, 1981] pratiqué pendant les inventaires selon la méthode décrite par Le Bail et al. [1981]. La fiabilité de cette technique est très proche de $100 \%$. Les mâles spermiants ont été mis en évidence par pression des flancs.

Avant leur remise à l'eau chaque poisson a été marqué au bleu alcian [Hart et Pitcher, 1969] sur une nageoire: pectorale droite pour les mâles spermiants, pelvienne droite pour les femelles maturantes et dorsale pour les autres. Ce marquage a été réalisé pour différencier ces individus, des géniteurs provenant du Scorff et marqués (pectorale gauche pour les mâles et pelvienne gauche pour les femelles) lors de leur capture dans le piège de montée pendant la migration de fraie.

\section{L'importance de la maturation sexuelle suivant l'âge}

Les sacrifices d'animaux étant exclus pendant la durée de l'étude à l'exception de 1983, nous avons abordé le problème de l'âge à la première maturation par l'intermédiaire de l'étude des marques de fraie sur les écailles.

Dans un premier temps les marques de fraie selon Backiel et Sych [1958] ont été recherchées sur des individus ayant maturé l'année précédente (marqués). Dans un deuxième temps nous les avons étudiées plus particulièrement chez les individus $4+$, de manière à établir à quel âge l'ensemble des individus sont matures.

\section{Le potentiel reproducteur}

La relation entre la fécondité absolue (F) et la longueur à la fourche (L) a été établie en Octobre 1983 après comptage des ovocytes sur un échantillon de 
21 femelles sacrifiées, et selon le modèle donné par Bagenal [1969]: $F=a L^{b}$.

Cette relation a servi par la suite aux calculs des différents potentiels reproducteurs (nombre d'ovules).

\section{Les tests statistiques}

La comparaison des distributions des effectifs a été faite à l'aide du Chi carré et la comparaison des moyennes par l'analyse de la variance.

\section{Resuitats}

La quasi totalité des géniteurs présents dans le ruisseau avant la migration de fraie sont sédentaires. Seuls quelques rares individus originaires de la rivière principale sont présents en octobre (Tableau 1). Après la fraie très peu de ces géniteurs sédentaires quittent le ruisseau (Tableau 2).

La présence de marque de fraie (Fig. 2) a été mise en évidence sur 121 des 131 poissons marqués (maturité vérifiée l'année précédente) examinés. L'étude de ces marques de fraie sur les écailles des truites $4+$ indique que la maturation sexuelle est générale chez les mâles comme chez les femelles à partir de $2+$ (Tableau 3). Compte tenu de la fiabilité de la technique de sexage utilisée, ce résultat nous permet de considérer que les individus âgés de plus de 2 ans n'ayant pas été détectés comme femelle maturante ou comme mâle spermiant sont des mâles non-spermiants. En conséquence, nous

Tableau 1. Présence dans la population en place (âges: $2+$, $3+$ et $4+$ ) de géniteurs venus de la rivière principale (marqués) lors de la migration de fraie de l'année précédente.

\begin{tabular}{lllll}
\hline & Mâles & & Femelles & \\
\cline { 2 - 3 } \cline { 5 - 6 } $\begin{array}{l}\text { Nombre } \\
\text { total }\end{array}$ & $\begin{array}{l}\text { Nombre } \\
\text { d'origine } \\
\text { rivière }\end{array}$ & $\begin{array}{l}\text { Nombre } \\
\text { total }\end{array}$ & $\begin{array}{l}\text { Nombre } \\
\text { d'origine } \\
\text { rivière }\end{array}$ \\
\hline 1981 & 169 & $1(0.6 \%)$ & 64 & $2(3.1 \%)$ \\
1982 & 182 & $1(0.5 \%)$ & 80 & $2(2.5 \%)$ \\
1983 & 157 & $2(1.3 \%)$ & 94 & $3(3.2 \%)$ \\
\hline
\end{tabular}

Tableau 2. Dévalaison des géniteurs du ruisseau (en place en Octobre) après la fraie.

\begin{tabular}{llllll}
\hline Hiver & Mâles & & \multicolumn{3}{l}{ Femelles } \\
\cline { 5 - 6 } \cline { 5 - 6 } & $\begin{array}{l}\text { Nombre } \\
\text { en }\end{array}$ & $\begin{array}{l}\text { Nombre } \\
\text { dévalant }\end{array}$ & $\begin{array}{l}\text { Nombre } \\
\text { en } \\
\end{array}$ & $\begin{array}{l}\text { Octobre } \\
\text { Octobre }\end{array}$ & $\begin{array}{l}\text { Nombre } \\
\text { dévalant }\end{array}$ \\
\hline $1980-1981$ & 395 & $7(1.8 \%)$ & 98 & $2(2.0 \%)$ \\
$1981-1982$ & 391 & $9(2.3 \%)$ & 100 & $1(1.0 \%)$ \\
$1982-1983$ & 389 & $2(0.5 \%)$ & 110 & $2(1.8 \%)$ \\
$1983-1984$ & 411 & $7(1.7 \%)$ & 143 & $1(0.7 \%)$ \\
\hline
\end{tabular}

avons calculé le taux de spermiation des mâles $2+$ et $3+$ au moment de chaque inventaire. $\mathrm{Ce}$ paramètre varie significativement suivant l'année en étant corrélé négativement à la température journalière des 2 semaines précédant l'inventaire, mais ne varie pas significativement suivant la classe d'âge ( $2+$ et $3+$ ) (Fig. 3). Ainsi, avons-nous considéré que cette observation était aussi valable pour la classe $1+$. Le nombre de mâles $1+$ maturants a donc été calculé à partir de celui des mâles $1+$ sper-

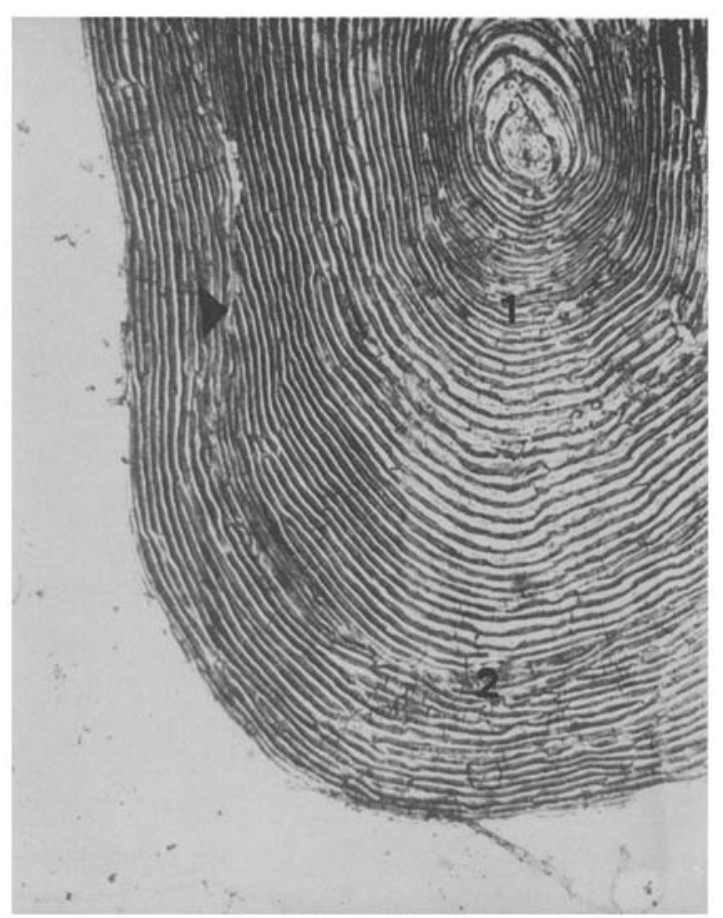

Fig. 2. Marque de fraie au niveau du 2ème hiver sur une écaille d'une truite femelle $2+$, détectée maturante à l'automne $1+$. 
Tableau 3. Etude des marques de 'fraie sur les écailles des poissons d'âge $4+\cdot M F 2, M F 3, M F 4$ : Marque de fraie au 2ème, 3ème, 4ème Hiver.

\begin{tabular}{lllll}
\hline & MF2 & MF3 & MF4 & Total \\
\hline Mâles & 19 & 23 & 23 & 23 \\
Femelles & 10 & 17 & 17 & 17 \\
Total & 29 & 40 & 40 & 40 \\
\hline
\end{tabular}

miants et de la moyenne des taux annuels de spermiation des mâles $2+$ et $3+$. Dans ces conditions le rapport des sexes chez les individus matures est toujours en faveur des mâles dont le nombre varie peu pendant la durée de l'étude, alors que celui des femelles augmente significativement. Les mâles sont en moyenne plus jeunes que les femelles, avec une forte proportion d'individus de la classe $1+(47$ à $62 \%$ du total). Chez les femelles la classe $2+$ est la mieux représentée ( 39 à $52 \%$ du total) et la classe $1+$ apporte environ $1 / 3$ de l'effectif. Les résultats des inventaires 1982 et 1983 montrent une augmentation du nombre de femelles $3+$ et surtout $4+$, ces dernières étant absentes en 1981 (Fig. 4).

Le taux de survie annuel pendant les 4ème et 5ème années d'existence peut être estimée à $30 \%$

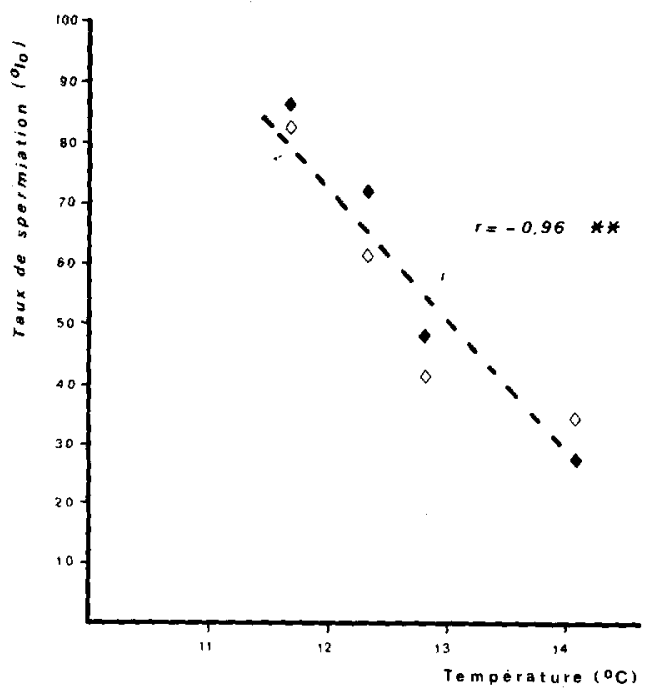

Fig. 3. Taux de spermiation des mâles $2+(\diamond)$ et $3+(\diamond)$ la 3 ème semaine d'octobre, en fonction de la température moyenne journalière des 2 semaines précédentes.

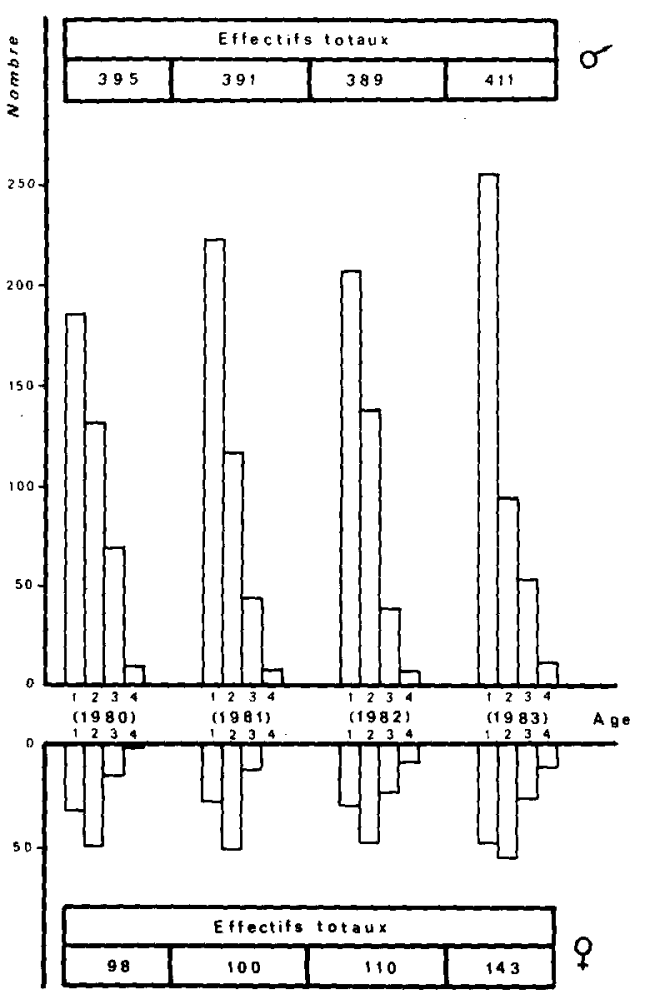

Fig. 4. Caractéristiques démographiques des géniteurs sédentaires.

chez les mâles matures et à $50 \%$ chez les femelles matures. Suivant les cohortes, le taux de survie tous sexes confondus au cours de la troisième année varie de 30 à $50 \%$.

Il n'y a pas de ségrégation spatiale entre les mâles et les femelles suivant la zone du ruisseau (Fig. 5).

La taille moyenne par classe d'âge varie significativement suivant l'année $(p=0.5 \%)$. En $1+$ les femelles matures sont plus grandes que les mâles spermiants eux-même plus grands que les immatures, en $2+$ cette différence de taille disparait (Fig. 6).

Le taux de maturation en $1+$ varie significativement selon l'année ( 44 à $77 \%$ ), il n'est pas corrélé à la taille moyenne des $1+$ mais à la taille moyenne des $0+$ l'automne précédant (Fig. 7).

La relation entre la fécondité absolue $(F)$ et la longueur à la fourche (L) calculée sur un échantillon de 21 femelles, de tailles comprises entre $136 \mathrm{~mm}$ et $260 \mathrm{~mm}$, est:

$\log F=1.64 \log L+1.042\left(r=0.755,{ }^{* *}\right)$ 

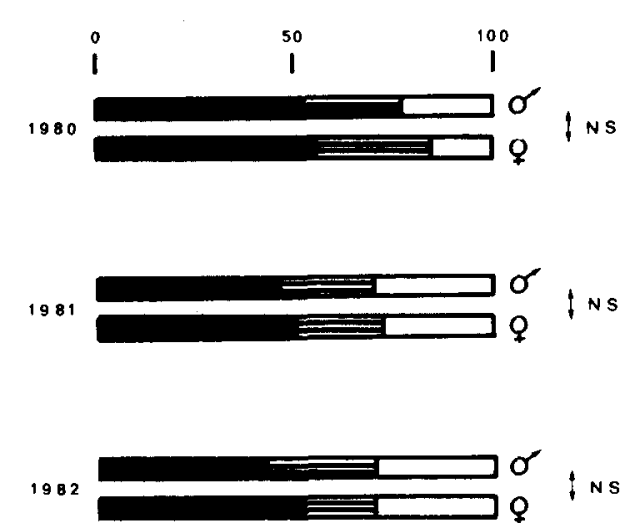

1983

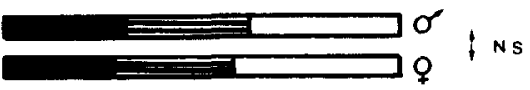

Fig. 5. Répartitions (en pourcentage) des mâles et des femelles suivant la zone.

Zone aval:

Zone moyenne:

Zone amont:
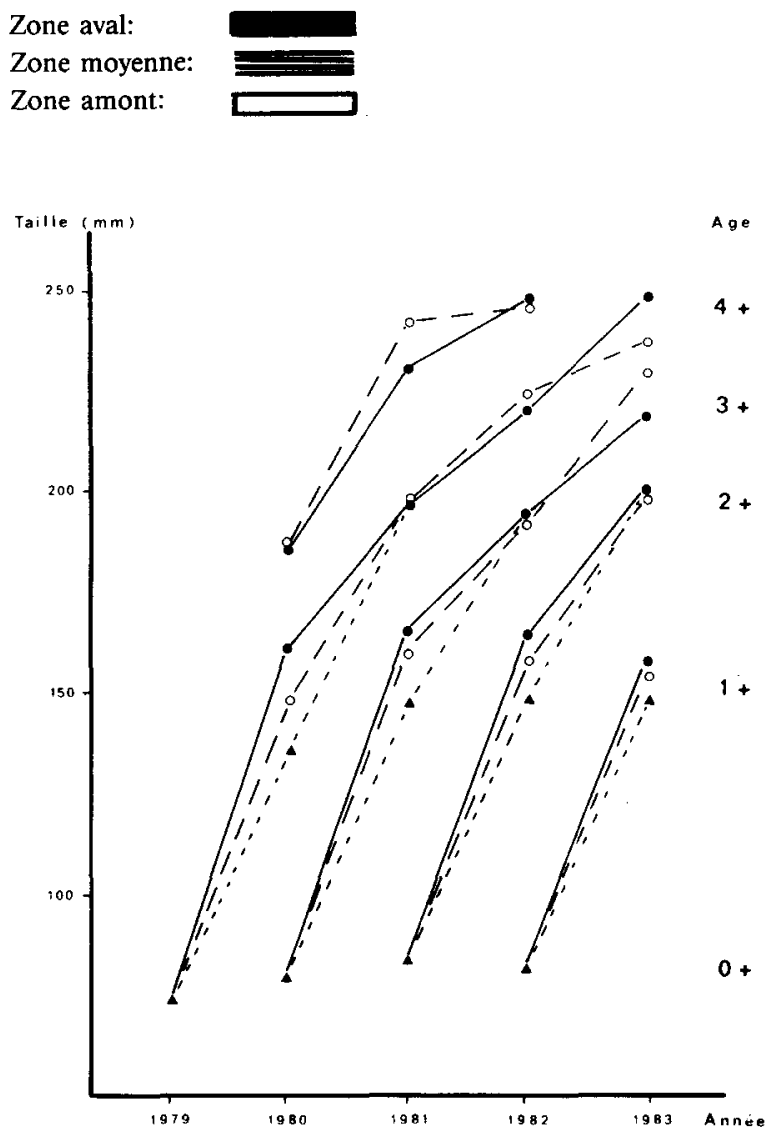

Fig. 6. Croissance moyenne des géniteurs sédentaires suivant le sexe. A Immatures; ○ mâles matures; - femelles matures.

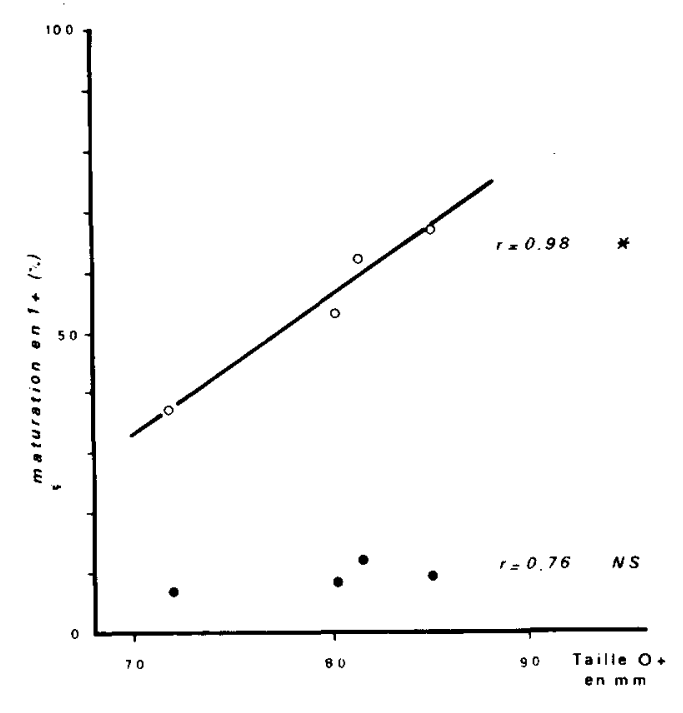

Fig. 7. Pourcentage de mâles matures $(0)$ et de femelles matures $(\bullet)$ chez les individus $1+$ en fonction de la taille moyenne des $0+$ l'automne précédent.

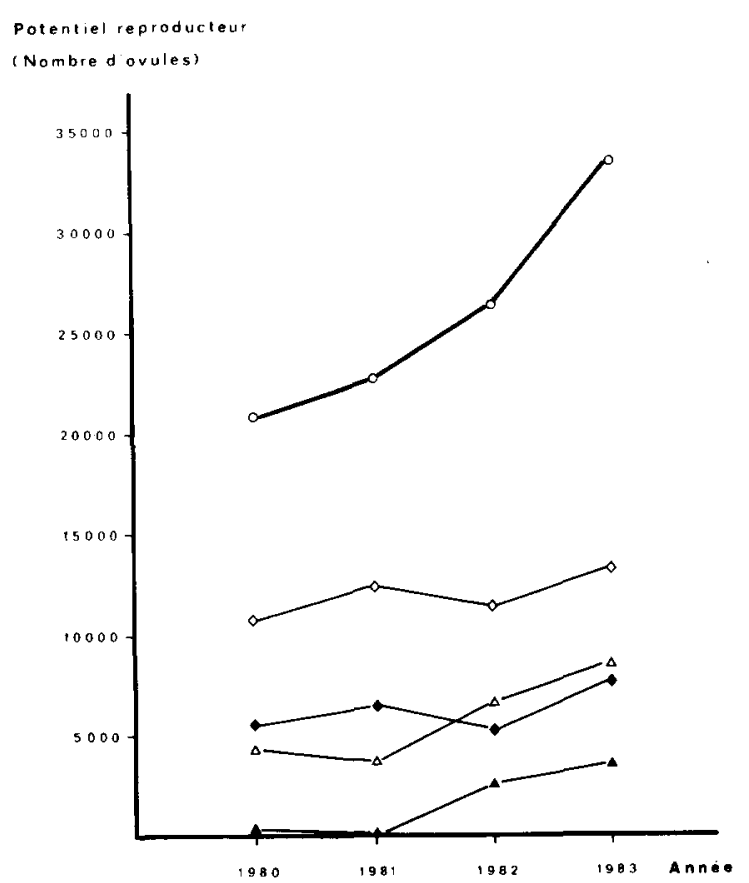

Fig. 8. Potential reproducteur annuel: Nombre d'ovules fournis par l'ensemble des femelles $(\circ)$, et les classes d'âge $1+(\bullet, 2+$ $(\diamond), 3+(\Delta)$, et $4+(\Delta)$. 
soit $F=0.0412 L^{1.64}$; avec $F$ en nombre d'ovules et $\mathrm{L}$ en $\mathrm{mm}$.

Etabli à partir de cette formule, le potentiel reproducteur total est en constante progression pendant la durée de l'étude, avec une participation des classes $3+$ et $4+$ en très nette augmentation à partir de 1982 (Fig. 8).

\section{Discussion}

Cette étude a démontré que la très grande majorité des géniteurs présents dans le ruisseau en octobre, y sont nés et y passeront toute leur existence.

Lensemble des géniteurs mâles et femelles est mûr en $2+$. Si la première maturation peut apparaitre en $0+$ chez quelques mâles isolés (observations personnelles), elle intervient de manière importante en $1+$, classe dans laquelle près de $80 \%$ des individus peuvent être matures [1982]. Cette précocité sexuelle de la population étudiée, par rapport aux populations de régions au climat moins tempéré [Alm, 1959; Horton, 1961; Mc Fadden et al., 1965; Lusk, 1968; Campbell, 1977; Jonsson, 1977] pourrait être en relation avec les bonnes conditions de croissance en $0+$, rencontrées sur le bassin du Scorff [Euzenat et Fournel, 1976; Baglinière, 1979]. En effet nos résultats montrent que les variations annuelles du taux de maturation en $1+$ sont corrélées à la croissance en $0+$, et non à la taille moyenne des truites $1+$, bien que, parmi ceux-ci, les individus matures soient en moyenne les plus grands, comme l'avait montré Alm [1959]. Cette influence de la croissance en $0+$ sur la maturation en $1+$ permet de comprendre pourquoi les truites $1+$ matures du bassin du Scorff peuvent avoir des tailles très différentes suivant le lieu. En 1980, la comparaison de nos résultats avec ceux d'autres études, que nous avons menées en parallèle sur la rivière principale et sur le ruisseau de Talascorn, affluent du ruisseau de Kernec (Fig. 1) montrent que la taille des femelles $1+$ matures varient considérablement suivant le lieu de capture, les intervalles de tailles se recoupant très peu (Tableau 4). La relation croissance-maturation selon Alm [1959] ne s'applique pas à l'ensemble des 3 populations mais à chacune prise séparément. En absence d'isole-
Tableau 4. Comparaison des tailles automnales des individus $\mathrm{O}+$ et $1+$ dans 3 milieux différents: le Scorff, le ruisseau de Kernec et le ruisseau de Talascorn

1 = longueur moyenne en $\mathrm{mm}$.

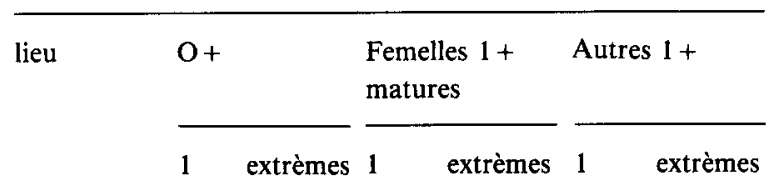

Scorff aval $\quad 95.3 \quad 73-114 \quad 186.5 \quad 180-235 \quad 169.4 \quad 142-235$

Ruisseau de

$\begin{array}{lllllll}\text { Kernec } & 80.0 & 51-118 & 160.7 & 140-182 & 139.9 & 100-225\end{array}$

Ruisseau de

$\begin{array}{llllllll}\text { Talascorn } & 68.1 & 52-83 & 129.3 & 106-148 & 119.6 & 101-148\end{array}$

ment génétique [Krieg \& Guyomard [1985] ont montré l'homogénéité génétique des truites du bassin du Scorff, pour 52 Loci étudiés] l'influence de la croissance sur l'âge à la lère maturation doit s'exprimer de la même manière dans les 3 populations. Les tailles des truites $0+$ s'inscrivent dans des intervalles se recoupant largement, il apparait que les facteurs de l'environnement rencontrés dans les 3 milieux sont moins discriminants pour la classe $0+$ que pour la classe $1+$. Les conditions de croissance en $0+$ peuvent dans les trois milieux, permettre à un certain nombre d'individus d'atteindre un seuil de taille suffisant pour que la maturation sexuelle se déclenche. Si, comme l'ont montré Sumpter et al. [1984] chez la Truite arc-en-ciel (Salmo gairdneri), les mécanismes physiologiques contrôlant la première maturation chez la femelle, se mettent en place au moins 1 an avant l'ovulation, ce seuil de taille se situerait à l'automne de la lère année. Par la suite, les facteurs de l'environnement, rencontrés dans les deux ruisseaux et la rivière principale, devenant plus limitants, la taille des truites $1+$ varie suivant le milieu, sans remettre en cause le processus de maturation déja engagé.

Cette hypothèse, d'une induction très précoce d'un phénomème biologique s'exprimant en $1+$, est semblable à celle proposée par Thorpe et al. [1980] pour la smoltification du Saumon atlantique (Salmo salar) et par Baglinière et Maisse [1985] pour la maturation précoce de cette même espèce.

Si les facteurs climatiques ont une action sur le potentiel reproducteur des $1+$ (nombre et taille des 
femelles maturantes) il semble que l'on puisse relier les variations du nombre de femelles des classes $3+$ et $4+$ aux facteurs humains. En 1980 et 1981 la pêche à la ligne était autorisée sur le ruisseau, elle fut interdite en 1982 et 1983.

La taille légale de capture fixée à $23 \mathrm{~cm}$, longueur totale, protège les $1+$ et la majorité de $2+$. Postérieurement à l'interdiction de la pêche on assiste à une augmentation du nombre de femelles $3+$ et surtout à l'apparition de femelles $4+$. Bien qu'aucune relation de cause à effet soit mise en évidence dans notre étude, l'hypothèse de l'influence de la pêche à la ligne sur la survie des femelles les plus âgées parait possible. Il est intéressant de noter que rien de comparable n'apparait chez les mâles. Cette différence de capturabilité pourrait être liée à une reprise du comportement alimentaire après la fraie, plus précoce chez les femelles, à une époque (Mars) où l'effort de pêche est le plus important sur les ruisseaux [Baglinière et al., 1979b]. Indépendamment de l'impact de la pêche à la ligne sur les femelles il est intéressant de noter que celles-ci ont un meilleur taux de survie annuel (environ 50\%) que les mâles (environ 30\%). Cette mortalité plus grande des mâles peut s'expliquer par une plus grande susceptibilité aux agents pathogènes en période de fraie [Pickering \& Christie, 1980]. Malgré cela le nombre total de mâles reste constant et ne constitue pas un facteur limitant dans la dynamique avec un rapport des sexes en leur faveur. On peut cependant, poser la question de l'influence sur la dynamique de la population de la relation température-spermiation. L'effet favorable des basses températures sur l'entrée en spermiation avait déjà été évoqué par Manning et Kime [1985] chez la Truite arc-en-ciel. Cette relation peut elle modifier le comportement de migration des mâles vers les zones de frayères? Les variations induites de la durée de stockage intra-testiculaire du sperme avant la fraie peuvent-elles avoir une influence sur la fécondance du sperme émis? Les conséquences éventuelles, sur la production de truites $0+$, des importantes variations du potentiel reproducteur seront discutées ultérieurement en relation avec l'apport des femelles migrantes, originaires du Scorff.

\section{Conclusion}

Cette étude, d'une durée limitée à 4 ans, a permis de mettre en évidence l'importance de la population de géniteurs sédentaires présents dans un ruisseau frayère avant qu'intervienne la migration de fraie des géniteurs de la rivière principale. Elle a permis de préciser l'importance de certains facteurs liés à l'environnement (température, capacités trophiques du milieu) ou aux activités humaines (pêche à la ligne) dans la dynamique de cette population.

Il apparait notamment que les conditions climatiques, de type océanique, sont à l'origine de la forte participation des femelles $1+$ au potentiel reproducteur $(25 \% \mathrm{du}$ total, soit l'équivalent de l'apport de la classe $3+$ ). Il est par contre difficile de porter un jugement de valeur sur l'impact de la pêche à la ligne. En effet en 1982 et 1983 l'apport de la classe $4+$ au potentiel reproducteur total reste limité à environ $10 \%$, et compte tenu de la possible prédation exercée par des individus de cette taille sur les 0+ [Mills, 1969] il n'est pas certain que l'influence de tels individus soit positive dans la dynamique de la population. Enfin il reste à préciser l'importance des géniteurs sédentaires dans la dynamique de la population de truites du ruisseau de Kernec, incluant la participation des géniteurs originaires de la rivière principale et le devenir des juvéniles produits. Ces deux aspects seront traités ultérieurement.

\section{Remerciements}

Cette étude a été réalisée dans le cadre d'une Convention INRA - Ministère de l'Urbanisme et du Logement no. 2527A.

La collaboration technique a été assurée par H. Peron.

Nous avons en outre bénéficié de l'aide des gardes pêche de la B.M.S. de la 2ème Délégation Régionale du C.S.P. et des étudiants de l'E.N.S. A. de Rennes. 


\section{References}

Alm, G., 1959. Connection between maturity, size and age in fishes. Institute of Freshwater Research Drottningholm, Report No. 40: 5-145.

Backiel, T. \& Sych, R., 1958. Scale resorption and spawning marks in sea trout (Salmo trutta L.) from Polish water. Rocz. Nauk. Roln 73: 119-148.

Bagenal, T. B., 1969. The relationship between food supply and fecundity in Brown trout Salmo trutta L. J. Fish Biol. 1: $167-182$.

Baglinière, J. L., 1979. Les principales populations de poissons sur une rivière à Salmonidés de Bretagne-sud, le Scorff. Cybium 3è série 7: 53-74.

Baglinière, J. L., A. Champigneulle \& A. Nihouarn, 1979a. La fraie du Saumon atlantique (Salmo salar L.) et de la Truite commune (Salmo trutta L.) sur le bassin du Scorff. Cybium 3è série 7: 75-96.

Baglinière, J. L., A. Nihouarn \& A. Champigneulle, 1979b. L'exploitation des salmonidés à la ligne sur le Scorff rivière de Bretagne sud. Bull. Fr. Piscic. 272: 94-115.

Baglinière, J. L. \& G. Maisse, 1985. Precocious maturation and smoltification in wild Atlantic Salmon in the Armorican massif, France. Aquaculture 45: 249-263.

Campbell, J. S., 1977. Spawning characteristics of brown trout and sea trout Salmo trutta L. in Kirk Burn, River Tweed, Scotland. J. Fish Biol. 11: 217-229.

Euzenat, G. \& F. Fournel, 1976. Recherches sur la truite commune (Salmo trutta L.) dans une rivière de Bretagne, le Scorff. Thèse 3è cycle Biol. anim. Fac. Sci. Univ. Rennes I, 213 pp.

Hart, P. J. B. \& T. J. Pitcher, 1969. Field trials of fish marking using a jet inoculator. J. Fish Biol. 1: 383-385.

Horton, P. A., 1961. The bionomics of brown trout in a dartmoor stream. J. Anim. Ecology 29: 311-338.

Huet, M. \& J. A. Timmermans, 1979. Fonctionnement et rôle d'un ruisseau frayère à truites. Station de Recherches Eaux et Forêts Groenendaal-Hoeilaart. Travaux - Série D, No. 48: 1 vol. $31 \mathrm{pp}$.

Jonsson, B., 1977. Demographic strategy in a brown trout population in western Norway. Zoologica Scripta 6: 255-263.

Krieg, F. \& R. Guyomard, 1985. Population genetics of French brown trout (Salmo trutta L.): large geographical differentiation of wild populations and high similarity of domesticated stocks. Génét. Sél. Evol. 17: 225-242.

Le Bail, P. Y. \& B. Breton, 1981. Rapid determination of the sex of puberal salmonid fish by a technique of immunoagglutination. Aquaculture 22: 367-375.
Le Bail, P. Y., G. Maisse \& B. Breton, 1981. Détection des femelles de salmonidés en vitellogenèse. 1. - Description de la méthode et mise en oeuvre pratique. Bull. Fr. Piscic. 283: $79-88$.

Libosvarsky, J., 1967. The spawning run of brown trout, Salmo trutta $\mathrm{m}$. fario L., and its analysis. Zoollogické Listy 16: $73-86$.

Lusk, S., 1968. Sexual maturity, sex ratio and fecundity in the Brown trout, Salmo trutta m. fario L., in the Loucka River. Zoologické Listy 17: 253-268.

Manning, N. J. \& D. E. Kime, 1985. The effect of temperature on testicular steroid production in the Rainbow trout, Salmo gairdneri, in vivo and in vitro. Gen. Comp. Endocrinol. 57: 377-382.

Mc Fadden, J. T., E. L. Cooper \& J. K. Andersen, 1965. Some effects of environment on egg production in Brown trout (Salmo trutta) Limnol. Oceanogr. 10: 88-95.

Mills, H. M., 1969. The survival of juvenile Atlantic salmon and Brown trout in some Scottish stream in 'symposium on salmon and Trout in streams' - H. R. Mac Millan. Lectures in Fisheries. University of British Columbia: 217-228.

Nihouarn, A., 1983. Etude de la Truite commune (Salmo trutta L.) dans le bassin du Scorff (Morbihan): démographie, reproduction, migrations. Thèse $3 \dot{e}$ cycle. Science Vie Envir. Univ. Rennes I, 72 pp.

Pickering, A. D. \& P. Christie, 1980. Sexual differences in the incidence and severity of ectoparasitic infestation of the brown trout, Salmo trutta L. J. Fish Biol. 16: 669-683.

Prouzet, P., 1981. Caractéristiques d'une population de Salmonidés (Salmo salar et Salmo trutta) remontant sur un affluent de l'Elorn (Rivière de Bretagne-Nord) pendant la période de reproduction 1979-1980. Bull. Fr. Piscic. 283: 140-154.

Seber, G. A. F. \& E. D. Lecren, 1967. Estimating population parameters from catches large relative to the population. J. Anim. Ecol. 36: 631-643.

Sumpter, J. P., A. P. Scott, S. M. Baynes \& P. R. Witthames, 1984. Early stage of the reproductive cycle in virgin female rainbow trout (Salmo gairdneri Richardson). Aquaculture 43: $235-242$.

Thorpe, J. E., R. I. G. Morgan, E. M. Ottaway \& M. S. Miles, 1980. Time of divergence of growth groups between potential $1+$ and $2+$ smolts among sibling Atlantic salmon. J. Fish. Biol. 17: 13-21.

Received 26 June 1986; accepted 24 November 1986. 